\title{
Echocardiographic evaluation of aorto-iliac occlusive disease
}

\author{
Grzegorz Styczynski • Cezary Szmigielski • \\ Anna Kaczynska · Jerzy Leszczynski • \\ Grzegorz Rosinski • Agnieszka Kuch-Wocial
}

Received: 29 July 2011 / Accepted: 10 October 2011/Published online: 19 October 2011

(C) The Author(s) 2011. This article is published with open access at Springerlink.com

\begin{abstract}
Several studies demonstrated feasibility of visual assessment of the common femoral artery Doppler waveform, in an indirect evaluation of aorto-iliac segment stenosis. Patients with cardiac diseases referred for echocardiography often have coexistent arterial pathology. Since many of them are potential candidates for endovascular procedures, we decided to study, whether echocardiography can be useful for detection of aorto-iliac occlusive disease. We evaluated 92 patients with abdominal aortic aneurysm or peripheral artery occlusive disease, referred from the vascular surgery department for cardiac evaluation before surgery. At the end of an echocardiographic examination, evaluation of flow in the distal external iliac arteries with an echocardiographic probe was performed. The Doppler waveform was classified into normal-with early diastolic flow
\end{abstract}

G. Styczynski ( $₫) \cdot$ C. Szmigielski · A. Kaczynska ·

A. Kuch-Wocial

Department of Internal Medicine, Hypertension and Angiology, Medical University of Warsaw,

Banacha 1a, 02-097 Warsaw, Poland

e-mail: grzegorz.styczynski@wum.edu.pl

\section{J. Leszczynski}

Department of General and Thoracic Surgery,

Medical University of Warsaw, Banacha 1a,

02-097 Warsaw, Poland

\section{G. Rosinski}

Department of Gastroenterology and Metabolic Diseases, Medical University of Warsaw, Banacha 1a,

02-097 Warsaw, Poland reversal or abnormal-without early diastolic flow reversal. Echocardiographic results were compared in a blinded fashion with reports from computed tomography angiography. Overall there were 58 iliac segments with significant $(\geq 70 \%)$ area stenosis or occlusion and 126 iliac segments without significant disease on computed tomography angiography. Abnormal Doppler waveform was found in 56 out of 58 abnormal iliac segments-sensitivity 97\%, and normal waveform was found in 106 out of 126 normal iliac segments-specificity $84 \%$. Positive predictive value of abnormal Doppler waveform for significant iliac disease was $74 \%$, and negative predicting value was $98 \%$. Detection of significant stenoses in aortoiliac segments is feasible with echocardiography. Further studies are necessary to evaluate its potential utility in a population of patients with cardiac disease referred for echocardiographic study.

Keywords Doppler echocardiography · Aorto-iliac occlusive disease $\cdot$ Screening

\section{Introduction}

Adult patients with cardiac diseases, referred for echocardiographic studies, often have coexistent arterial pathology. It has been demonstrated that coronary artery disease (CAD) and degenerative aortic stenosis, are associated with increased prevalence of peripheral artery occlusive disease (PAOD) [1-7]. Significant 
aorto-iliac occlusive disease is estimated to be present in about half of the PAOD population [8]. If feasible, evaluation of this arterial segment, as an extension of a routine echocardiographic study, could provide important additional diagnostic and prognostic information. Several studies showed the possibility of indirect evaluation of aorto-iliac stenoses by visual assessment of Doppler waveform in common femoral arteries, registered with a vascular high frequency probe. However, diagnostic accuracy of this method differed significantly between studies. When arteriography was used as a reference method, visual assessment of Doppler waveform in the common femoral arteries had high sensitivity, specificity, positive and negative predictive value for significant aorto-iliac stenoses or occlusions [9, 10]. However, in studies that used MR angiography as a reference method, the results were reported to be significantly worse [11, 12].

As the distal part of the external iliac artery has superficial course and is easily visualized in the inguinal area using an echo probe, we decided to check the value of the visual assessment of Doppler waveform in predicting significant aorto-iliac occlusive disease.

\section{Methods}

\section{Patients}

We studied 92 patients referred from the vascular surgery department for a cardiac evaluation before surgery. Forty two patients had abdominal aortic aneurysm (AAA) and 50 patients had peripheral artery occlusive disease (PAOD) as the main reason for hospitalization. Twelve patients with AAA had concomitant PAOD. Most of the patients had typical risk factors for atherosclerosis and were treated with various drugs including antihypertensives, aspirin and statins. Characteristics of the patients is presented in Table 1. The study was accepted by the local Ethics Committee and the patients gave informed consent for echocardiographic and vascular examinations.

Echocardiographic evaluation

At the end of an echocardiographic examination (GE/Vingmed System Five scanner), evaluation of the flow in the distal external iliac arteries with an
Table 1 Characteristics of patients

\begin{tabular}{lll}
\hline Variable & $\begin{array}{l}\text { PAOD present } \\
\mathrm{N}=62\end{array}$ & $\begin{array}{l}\text { PAOD absent } \\
\mathrm{N}=30\end{array}$ \\
\hline Age & $64 \pm 11$ & $69 \pm 8$ \\
Sex (F/M) & $15 / 47$ & $2 / 30$ \\
BMI & $25 \pm 4$ & $27 \pm 3$ \\
ABI & $0.57 \pm 0.15$ & $1.03 \pm 0.07$ \\
Fontaine functional class & & \\
I (n) & 0 & 30 \\
IIa (n) & 9 & 0 \\
IIb (n) & 21 & 0 \\
III (n) & 17 & 0 \\
IV (n) & 15 & 0 \\
Aorto-iliac PAOD (n) & 40 & 0 \\
Infrainguinal PAOD (n) & 22 & 0 \\
Smoking (\%) & 66 & 53 \\
Arterial hypertension (\%) & 80 & 70 \\
Hypercholesterolemia (\%) & 29 & 33 \\
Diabetes (\%) & 42 & 17 \\
\hline PAOD peripheral arter & &
\end{tabular}

$P A O D$ peripheral artery occlusive disease, $B M I$ body mass index, $A B I$ ankle-brachial index

echocardiographic probe was performed. The patients were placed in the supine position and the external iliac arteries (EIA) were localized in the inguinal area, first with two-dimensional, and than with color Doppler imaging (Fig. 1). Usually both external iliac vein and artery are seen close together and the artery is easily differentiated by the pulsatile character of the flow. The course of the distal EIA is almost parallel to the Doppler line. The pulse wave Doppler flow recordings were made with the same sample size used in echocardiographic study and stored on a video tape or on a digital system (Echopac, GE). The EIA Doppler waveform was classified into normal-with early diastolic flow reversal (Fig. 2) or abnormalwithout early diastolic flow reversal (Fig. 3). EIA Doppler waveform recordings were performed by an experienced echocardiographer aware of surgical diagnosis but blinded to the level of arterial disease. Analysis of the waveforms was performed off-line by another experienced echocardiographer blinded to the patients' diagnosis and level of arterial disease.

Angiographic evaluation

Evaluation of the presence and level of arterial disease was based on the reports from computed tomography 


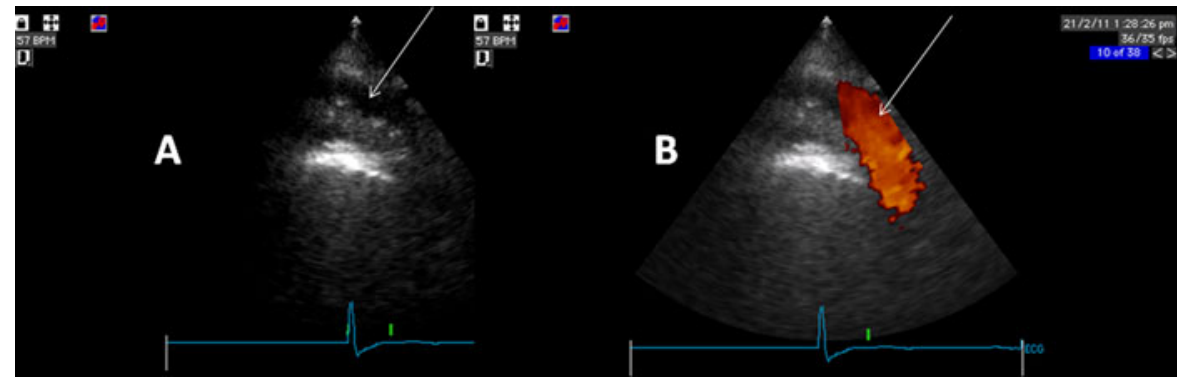

Fig. 1 External iliac artery seen on 2D image (a-arrow) and with color Doppler (b-arrow)

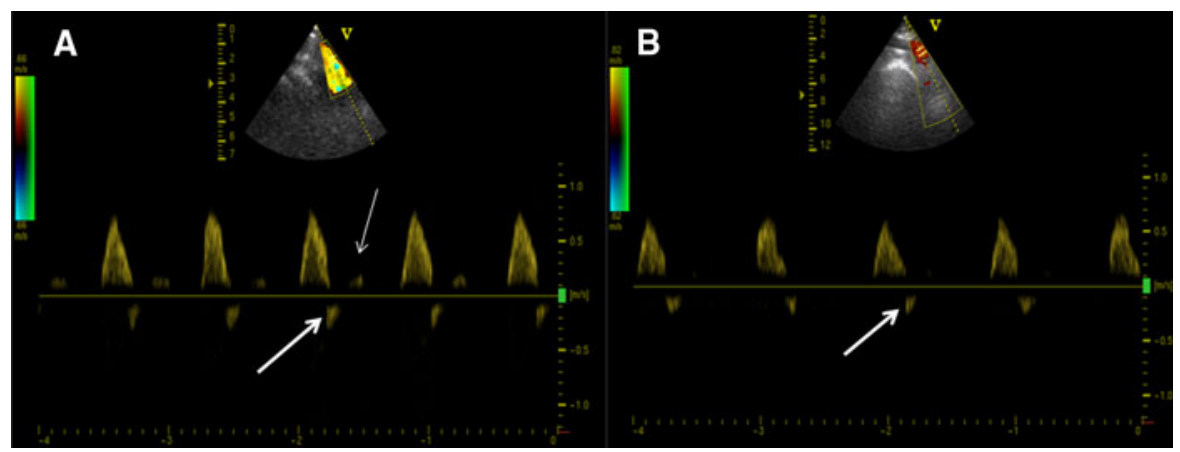

Fig. 2 a Normal triphasic flow in the distal external iliac artery with early diastolic flow reversal (thick arrow) and diastolic forward flow (thin arrow). b Normal biphasic flow in the external iliac artery registered with echocardiographic probe with early flow reversal (thick arrow)

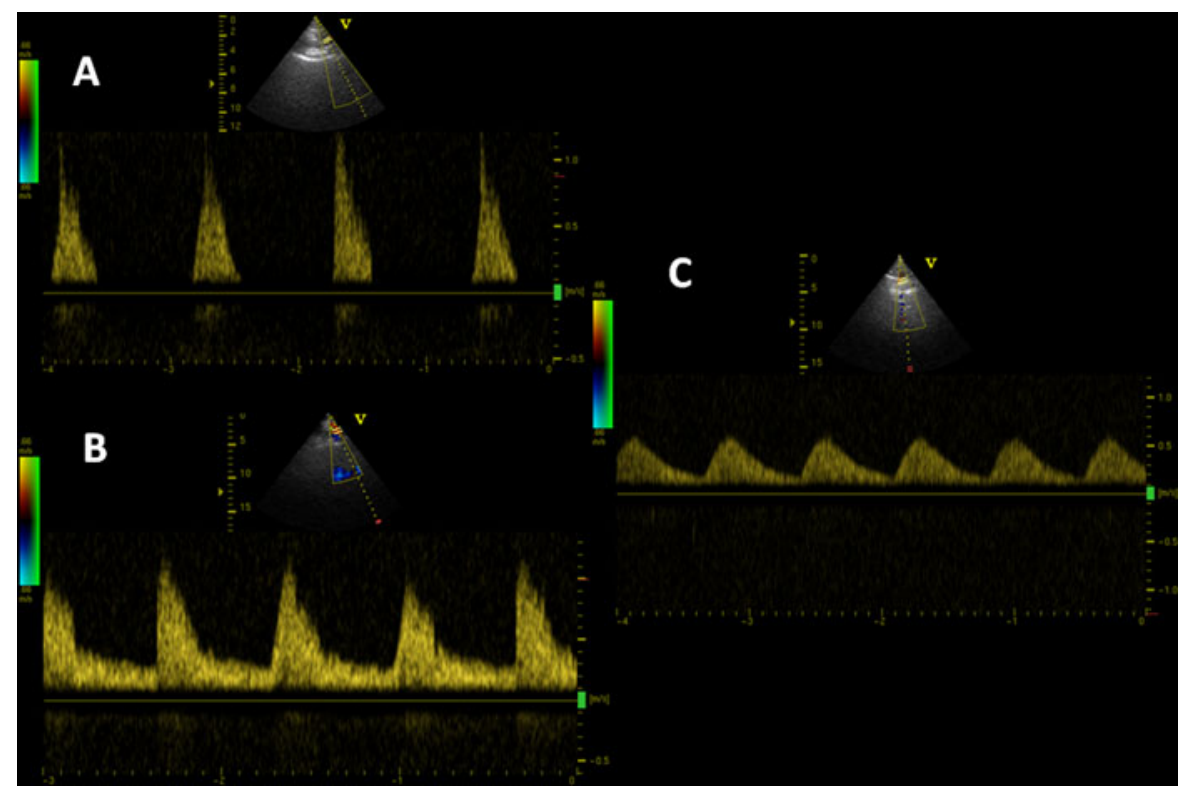

Fig. 3 a Abnormal Doppler waveform in the distal external iliac artery characterized by sharp and tall systolic phase and lack of diastolic flow reversal. b Abnormal Doppler waveform in the distal external iliac artery characterized by sharp and tall systolic phase, lack of diastolic flow reversal and presence of diastolic forward flow. c Abnormal Doppler waveform in the distal external iliac artery characterized by blunted systolic phase, lack of diastolic flow reversal and presence of diastolic forward flow-typical for proximal occlusion 
angiography (LightSpeed 16, GE). Significant aortoiliac disease was defined as presence of $\geq 70 \%$ area stenosis or occlusion in the aortoiliac segment.

\section{Statistical analysis}

Sensitivity was calculated according to the formula: number of true positive cases (i.e. cases with abnormal waveform in patients with aortoiliac disease)/number of true positive + false negative cases (i.e. cases with normal waveform in patients with aortoiliac disease). Specificity was calculated as a number of true negative cases (i.e. cases with normal waveform in patients without aortoiliac disease)/number of true negative + false positive cases (i.e. cases with abnormal waveform in patients without aortoiliac disease). Positive predictive value (PPV) was calculated as a number of true positive cases/number of true positive + false positive cases. Negative predictive value (NPV) was calculated as a number of true negative cases/number of true negative + false negative cases. Interobserver agreement on the presence of notch was evaluated with kappa statistics [Kappa $=($ Observed agreement-Chance agreement $) /(1-$ Chance agreement)], based on the assessment of the first 50 consecutive patients by 2 echocardiographers. Continuous variables are presented as mean \pm standard deviation.

\section{Results}

Out of 92 patients 40 had significant stenosis or occlusion in the aorto-iliac segment. Among them, 18 had aortic or bilateral iliac disease, and 22 unilateral iliac disease. Fifty two patients had no significant aorto-iliac disease, and in this group, 22 patients had distal (infrainguinal) PAOD, and 30 patients had no PAOD. Overall there were 58 iliac segments with significant stenosis or occlusion and 126 iliac segments without significant disease. Technically adequate registration of distal EIA Doppler waveform was possible in all patients. Abnormal Doppler waveforms were found in 56 out of 58 abnormal iliac segmentssensitivity $97 \%$, and normal waveforms were found in 106 out of 126 normal iliac segments-specificity $84 \%$. The positive predictive value (PPV) of abnormal Doppler waveform for significant iliac disease was $74 \%$, and the negative predicting value (NPV) $98 \%$. However, among 20 false positive waveforms regarding iliac segments, there were 17 segments with superficial femoral artery occlusion. Thus, inclusion of these arterial segments would reduce false positive abnormal waveforms to 3 and increase PPV to $96 \%$. Interobserver agreement in the classification of EIA waveforms into normal or abnormal was very good, with kappa value $=0.847$. Mean prolongation of echocardiographic study, based on the analysis of the first 30 measurements, was only $3.1 \pm 0.9 \mathrm{~min}$.

\section{Discussion}

Results of our study show, that detection of the significant stenoses in aorto-iliac segments by the distal EIA Doppler waveform evaluation is feasible with echocardiography. The distal EIA, that continues into common femoral artery, is a large vessel that lies superficially in the inguinal area and can be easily identified with 2D and color Doppler imaging. The spectral Doppler signal is usually of very good quality. In our study, visualization of the distal EIA with color Doppler and interpretation of the spectral Doppler signal was possible in all subjects. Visual interpretation of the EIA Doppler waveforms regarding the presence or absence of the early diastolic flow reversal was also easy, resulting in a high interobserver agreement between 2 echocardiographers-one of them unexperienced in peripheral vascular ultrasound. Thus we believe that distal EIA Doppler waveform acquisition and analysis should not be problematic for practising echocardiographers.

An absence of diastolic flow reversal in the Doppler waveform is a well-established indicator of significant lower extremity arterial stenosis [13-16]. In our study, this simple measure had good diagnostic performance in the evaluation of aorto-iliac occlusive disease. The presence of diastolic reversal (normal waveform) in the distal EIA almost always excluded significant aorto-iliac stenosis. However, absence of the diastolic reversal (abnormal waveform) was present in both aorto-iliac and superficial femoral artery (SFA) stenoses, resulting in lower positive predictive value regarding aorto-iliac segment. We believe that this is due to the phenomenon of Doppler waveform distortion not only distally, but also proximally to a significant stenosis [17]. In one study, a specific abnormal Doppler waveform, that was registered in the common femoral artery, was predictive of SFA 


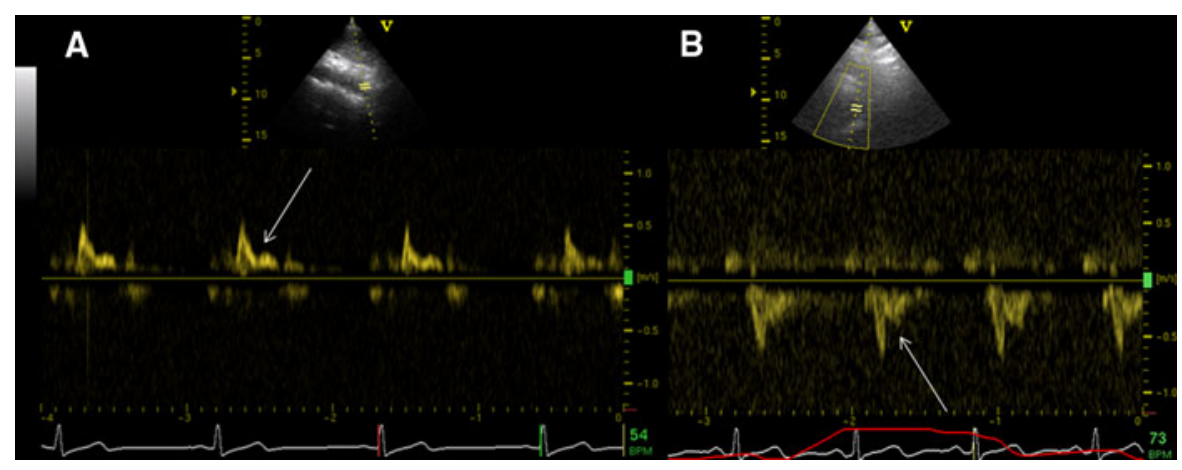

Fig. 4 a Mid-systolic deceleration "notch" in abdominal aortic Doppler waveform—arrow. b Mid-systolic deceleration "notch" in descending aortic Doppler waveform-arrow

stenosis [11]. We speculate that in a significant proximal SFA occlusive disease, an abnormal Doppler waveform, registered in the distal EIA, may be due to the proximity of both vessels.

We previously reported another echocardiographic Doppler finding, that can be helpful in the diagnosis of aorto-iliac occlusive disease $[18,19]$. In these patients, characteristic mid-systolic deceleration "notch" in the Doppler waveform, registered in the proximal descending or abdominal aorta is often present (Fig. 4). Although diagnostic performance of this simple evaluation is reliable, with sensitivity and specificity over $80 \%$, we believe that it is still inferior to the distal EIA assessment for several reasons. It cannot localize side of a stenosis. In some patients, adequate Doppler evaluation of the abdominal or proximal descending aorta is impossible due to poor acoustic window. Additionally, the interobserver agreement on the midsystolic notch diagnosis may be worse than that for the EIA waveform interpretation. Moreover, the presence of notch (which results from high amplitude backward pressure wave reflection from occlusions or significant stenoses in aorto-iliac segment) may be influenced by many factors that also influence aortic pulse wave velocity i.e. age, arterial wall stiffness, blood pressure, heart rate, arterial diameter and blood density.

Evaluation of aorto-iliac occlusive disease is potentially attractive in the echocardiographic laboratory, as significant number of patients with atherosclerotic cardiac disease, referred for echocardiography have coexistent peripheral artery occlusive disease [1-7]. In this population information about aorto-iliac segment may have important diagnostic and prognostic significance. For example, many patients with coronary artery disease, and aortic stenosis, are potential candidates for endovascular intervention, and echocardiographic assessment of aorto-iliac segment may be helpful for the choice of the vascular access. However, potential value of this method should be tested on broad "echocardiographic" population, that differs from our selected group of the vascular surgery patients.

\section{Limitations}

Although digital subtraction angiography is regarded as a gold standard for arterial evaluation, CT angiography is considered as an acceptable alternative for vascular study. In our group of patients CT studies were interpreted by experienced radiologists, however, no comparisons for interobserver agreement were available.

In our study, patients with PAOD presented with severe symptoms and most of them had occlusions or high-grade arterial stenosis. It is unclear whether diagnostic performance of the EIA Doppler waveform analysis would be similar in patients with less advanced disease.

We did not compare the diagnostic value of EIA Doppler analysis over femoral pulse palpation. Based on the data from literature, we assumed that physical examination is not sufficiently reliable for evaluation of aorto-iliac stenoses [20, 21].

\section{Conclusion}

Evaluation of aorto-iliac occlusive disease is feasible with echocardiography. Further studies are necessary to confirm its potential utility in populations of 
patients with different cardiac diseases referred for echocardiographic study.

\section{Conflict of interest None.}

Open Access This article is distributed under the terms of the Creative Commons Attribution Noncommercial License which permits any noncommercial use, distribution, and reproduction in any medium, provided the original author(s) and source are credited.

\section{References}

1. Przewłocki T, Kabłak-Ziembicka A, Kozanecki A et al (2009) Polyvascular extracoronary atherosclerotic disease in patients with coronary artery disease. Kardiol Pol 67:978-984

2. Steg G, Bhatt D, Wilson P et al (2007) One-year cardiovascular event rates in outpatients with atherothrombosis. JAMA 297:1197-1206

3. Makowsky M, McAlister F, Galbraight D et al (2008) Lower extremity peripheral arterial disease in individuals with coronary artery disease: prognostic importance, care gaps, and impact of therapy. Am Heart J 155:348-355

4. Dieter R, Tomasson J, Gudjonsson T et al (2003) Lower extremity peripheral artery disease in hospitalized patients with coronary artery disease. Vasc Med 8:233-236

5. Poredos P, Jug B (2007) The prevalence of peripheral artery disease in high risk subjects and coronary or cerebrovascular patients. Angiology 58:309-315

6. Aronow W, Ahn C, Kronzon I (2001) Association of valvular aortic stenosis with symptomatic peripheral arterial disease in older persons. Am J Cardiol 88:1046-1047

7. Rigatelli G (2004) Aortoiliac angiography during coronary artery angiography detects significant occult aortoiliac and renal artery atherosclerosis in patients with coronary atherosclerosis. Int J Cardiovasc Imaging 20:299-303

8. Aboyans V, Desormais I, Lacroix P, Salazar J, Criqui MH, Laskar M (2010) The general prognosis of patients with peripheral arterial disease differs according to the disease localization. J Am Coll Cardiol 55:898-903

9. Eiberg J, Jensen F, Gronval Rasmussen J, Schroeder T (2001) Sreening for aortoiliac lesions by visual interpretation of the common femoral Doppler waveform. Eur J Vasc Endovasc Surg 22:331-336
10. Shaalan W, French-Sherry E, Castilla M, Lozanski L, Bassiouny H (2003) Reliability of common femoral artery hemodynamics in assessing the severity of aortoiliac inflow disease. J Vasc Surg 37:960-969

11. Spronk S, den Hoed P, de Jonge L, van Dijk L, Pattynama P (2005) Value of the duplex waveform at the common femoral artery for diagnosing obstructive aortoiliac disease. J Vasc Surg 42:236-242

12. O`Neill S, George R, Wallace W, Blair P, McKinley A (2011) Do Doppler waveforms at the common femoral artery accurately predict iliac stenosis? Ir J Med Sci 180:247-249

13. Kohler T, Nance D, Cramer M, Vandenburghe N, Strandness D (1987) Duplex scanning for diagnosis of aortoiliac and femoropopliteal disease: a prospective study. Circulation 76:1074-1080

14. Jager K, Philips D, Martin R et al (1985) Noninvasive mapping of lower limb arterial lesions. Ultrasound Med Biol 11:515-521

15. De Smet A, Ermers E, Kitslaar P (1996) Duplex velocity characteristics of aortoiliac stenoses. J Vasc Surg 23: 628-636

16. Walton R, Martin T (1984) Prospective assessment of the aorto-iliac segment by visual interpretation of frequency analysed Doppler waveforms-a comparison with arteriography. Ultrasound Med Biol 10:27-32

17. Schaberle W (2011) Peripheral arteries. In: Schaberle W (ed) Ultrasonography in vascular diagnosis-a therapy oriented textbook and atlas, 2nd edn. Springer, Berlin, pp 60-67

18. Styczynski G, Szmigielski C, Leszczynski J, Abramczyk P, Kuch-Wocial A, Szulc M (2009) Descending aortic Doppler flow pattern in patients with proximal peripheral artery disease. Am J Cardiol 103:1774-1776

19. Styczynski G, Szmigielski C, Leszczynski J, Kuch-Wocial A, Szulc M (2010) Abdominal aortic Doppler waveform in patients with aorto-iliac disease. Eur J Vasc Endovasc Surg 39:714-718

20. Brearley S, Schearman C, Simms M (1992) Peripheral pulse palpation: an unreliable physical sign. Ann R Coll Surg Engl 74:169-171

21. Myers K, Scott D, Devine T, Johnston A, Denton M, Gilfillan I (1987) Palpation of the femoral and popliteal pulses: a study of the accuracy as assessed by agreement between multiple observers. Eur J Vasc Surg 1:245-249 